\title{
VARIACIÓN ALOFÓNICA Y DIATÓPICA DEL FONEMA / b / EN EL ESPAÑOL DE PROFESIONALES CHILENOS EN SITUACIÓN DE LECTURA EN VOZ ALTA*
}

\author{
Jaime Soto-Barba** \\ Erika Díaz ${ }^{* * *}$ \\ Daniel Ignacio Pereira ${ }^{* * * *}$
}

\section{Resumen}

En este trabajo, se aportan datos sobre la variación fonética y diatópica del fonema /b/ en el habla de profesionales chilenos. Los resultados muestran la presencia de cinco variantes del fonema $/ \mathrm{b} /$, aunque solo tres de ellas fueron relevantes estadísticamente: $[\mathrm{b}],[\beta]$ y [0]. De estas últimas, la variante oclusiva [b] fue la más estable. La aproximante bilabial [B] predomina a nivel país, seguida de la aproximante labiodental [ט]. Los datos sugieren una variación dialectal del labiodentalismo entre las zonas norte y centro, y las zonas sur y sur austral.

Palabras clave: Variación de /b/, labiodentalismo, fonética articulatoria, lectura en voz alta.

\section{ALLOPHONIC AND DIATOPIC VARIATION OF THE SPANISH / b/ PHONEME OF CHILEAN PROFESSIONALS IN READING ALOUD CONTEXT}

\begin{abstract}
In this study, data on the phonetic and diatopic variation of $/ \mathrm{b} /$ phoneme produced by Chilean professionals is presented. The results showed five variants of $/ \mathrm{b} /$, although only three were statistically significant: $[b],[\beta]$ y $[0]$. Of these three, the plosive variant $[b]$ was the most stable. The bilabial approximant $[\beta]$ predominates across the country followed by the labiodental approximant [0]. These results also suggest a dialectal variation of the labiodentalism between northen and center areas, and between southern and southernmost zones.
\end{abstract}

Key words: Variation of /b/, labiodentalism, articulatory phonetics, reading aloud.

* Este artículo forma parte de los resultados del proyecto FONDECYT Regular No1120886, denominado "Descripción fonético-fonológica de un modelo de pronunciación enseñable del español culto de Chile".

** Chileno. Doctor en Lingüística. Académico Universidad de Concepción, Concepción, Chile. jstot@ udec.cl

*** Chilena. Licenciada en Educación. Profesora de Español. Universidad de Concepción, Concepción, Chile.erikadiaz@udec.cl

**** Chileno. Doctor en Lingüística. Académico Universidad de Concepción, Concepción, Chile. danielpereira@udec.cl 


\section{Introducción}

Los estudios fonético-fonológicos que han observado la variación del fonema / $b$ / en el español de Chile no exploran, en general, las tendencias globales sobre el real comportamiento fonológico de este segmento. Mucho menos se cuenta con un estudio que oriente sus principales variaciones fonéticas hacia la enseñanza de la pronunciación del español chileno.

Esto último resulta de vital importancia, pues es sabido que los profesores que ejercen docencia en la enseñanza básica continúan instruyendo a los niños que los grafemas $<\mathrm{v}>\mathrm{y}<\mathrm{b}>$ tienen una realización fonética diferenciada (labiodental, el primero; bilabial, el segundo), con lo que se fomenta una distinción fonémica que hace bastante tiempo dejó de existir en la lengua castellana.

Según Sadowsky (2010), existe un rechazo generalizado hacia la posibilidad de que en español el fono labiodental sonoro [v] sea un alófono del fonema /b/. En este punto, resulta muy útil recordar los planteamientos de Navarro Tomás (1932), quien señala que no existe noticia de que la variante labiodental [v] haya sido corriente en la pronunciación española. Para este autor, entre los españoles ${ }^{1}$ solo pronuncian [v] por $<\mathrm{v}>$ las personas demasiado influidas por prejuicios ortográficos o particularmente propensas a afectación o por influencia de otras lenguas (variedades del catalán o inglés, por ejemplo). Esta idea podría explicar la manera particular con que en Chile se produce el fonema bilabial /b/: probablemente, quienes pronuncian [v] lo hacen motivados por el español público de Chile, en el que, de acuerdo con Salas (1996-1997), se presenta esta pronunciación, o por hipercorrección ortográfica, la que consiste en distinguir fonéticamente la pronunciación de los grafemas $<\mathrm{b}>\mathrm{y}<\mathrm{v}>$.

Lenz (1893 y 1909) señala que la pronunciación normal de los grafemas $<\mathrm{b}>\mathrm{y}<\mathrm{v}>$ en Chile es [ $\beta$ ] , especialmente, entre vocales y después fonemas laterales; mientras que precedidas de nasal se pronuncia como [b]; en cambio, en posición inicial absoluta, $[\mathrm{b}]$ y $\left[\beta_{\mathrm{r}}\right]$ alternan. En definitiva,

1 Entiéndase aquí como gentilicio, no como término para referirse a los hablantes de español. 
Lenz concluye que ambos grafemas representan el mismo sonido, lo que autoriza la afirmación de Sawdosky (2010).

También Saavedra (1906, referido en Ortiz y Saavedra, 2003: 37) indicó que "en la pronunciación chilena de / b-d-g/ en posición intervocálica no existe contacto entre los articuladores", por lo que el modo de articulación de /b/ sería fricativo y su estatus estaría a medio camino entre estas y las vocales.

Posteriormente, Wigdorsky (1978) y Wigdorsky (1983, referido en Ortiz y Saavedra, 2003: 46-47), en sus estudios sobre las diferencias entre hablantes cultos y no cultos, junto con afirmar la ausencia de diferencias significativas entre estos grupos, establece que existe una marcada tendencia a elidir /b/ en posición intervocálica, en desmedro de $[\beta]$. Señala también que el uso general es realizar el fonema /b/ como [b] ante / s, t/, como en el caso de lapso ['labso] y apto ['abto].

Cepeda (1991 y 1994) y Cepeda y Poblete (1993), en sus estudios sobre el español hablado en Valdivia, apunta a resultados similares a los descritos anteriormente. Por ejemplo, en el trabajo de 1991, Cepeda observa la presencia de un alófono $[\mathrm{v}]$ para / b/, aunque considera que es frecuente en las mujeres de estrato sociocultural alto, posiblemente por influjo del adstrato alemán ${ }^{2}$ y con un claro rasgo de prestigio.

A raíz de lo anterior, Sadowsky (2010) indica que existen por lo menos tres estudios que consideran que la variante labiodental [v] es una alternativa alofónica a la pronunciación del fonema /b/: el de Cepeda (1991) ya comentado arriba; el de Cartagena (2002), que sitúa la presencia de la variante labiodental en la pronunciación familiar y en el habla cuidada, asociada a la distinción de los grafemas $\langle\mathrm{b}\rangle \mathrm{y}<\mathrm{v}\rangle$; y el estudio de Borland Delorme (2004:106), sobre la variación alofónica de la norma culta de Santiago de Chile. En su artículo, Sadowsky concluye que [v] es el alófono dominante ${ }^{3}$ de la pronunciación de $/ \mathrm{b} /$, tanto por la cantidad de veces que se repite en su corpus (59,5\% de realizaciones), como por el número de contextos fonéticos en que aparece.

2 Bajo los gobiernos de Manuel Bulnes y Vicente Pérez Rosales (siglo XIX) se impulsó la llamada Ley de Colonización, la que entregaba estímulos a inmigrantes extranjeros para asentarse en distintos lugares del sur de Chile. La zona de Valdivia y Llanquihue fue colonizada, precisamente, por ciudadanos alemanes. (cf. http: / / www.memoriachilena.cl/602/w3-article-676.html)

3 En su artículo, Sadowsky (2010) clasifica los fonos [v] y [v] dentro de la categoría [v], pues le interesa observar el punto y no el modo de articulación de las variantes de /b/. Esto es importante señalarlo, pues incide en las conclusiones de esta investigación. 
Más recientemente, Soto-Barba (2011) señala que la selección de las variantes del fonema bilabial /b/ está constreñida por contexto fonético, en cuyo caso pueden manifestarse las siguientes variaciones: [b] bilabial oclusivo sonoro oral en posición inicial absoluta y precedido de consonante nasal; y $\left[\beta_{\uparrow}\right]$, bilabial aproximante sonoro oral en los demás contextos. Además, demuesta que la variación también podría explicarse diastráticamente, pues observa diferencias en la pronunciación de hablantes urbanos de nivel socio-cultural alto y bajo y hablantes rurales de nivel sociocultural bajo. Esto se evidencia en que en los primeros se manifiestan dos variantes: una [v] fricativa labiodental sonora oral y, sobre todo, una [0] aproximante labiodental sonora oral, variantes que no aparecieron en los otros estratos.

Finalmente, Vergara (2011) estudió el comportamiento del fono fricativo labiodental sonoro [v], en una población de preescolares de la provincia de Concepción-Chile, y luego, en Vergara y Pérez (2013) hace lo mismo, pero en un grupo de estudiantes de secundaria con la misma procedencia. Esta doble observación le permite comprobar si el aprendizaje de la lectoescritura es el responsable de dicha pronunciación. Tras analizar sus enunciados, constató que el alófono fricativo labiodental sonoro [v] es la realización más frecuente del fonema /b/, en consonancia con lo expuesto por Sadowsky (2010), y que aparece tanto en sujetos alfabetizados como en aquellos que aún no comienzan este proceso de enseñanza formal, por lo que descarta la influencia de los grafemas $<b>$ $\mathrm{y}<\mathrm{v}>$ en la aparición de la realización labiodental. Esto implica que el esfuerzo didáctico de los profesores en hacer la distinción no tiene efecto en hablantes que se han sometido a la educación formal.

En síntesis, la revisión bibliográfica muestra que, si bien existen estudios que observan el comportamiento fonético-fonológico del fonema / b / tanto en Santiago, como en Valdivia, Chillán y Concepción, hace falta una discusión y análisis en torno a su distribución alofónica y diatópica a lo largo del territorio nacional, sobre todo considerando los porcentajes de frecuencia que aportan los artículos revisados y la nula existencia de muestras de habla del norte del país, donde el contacto con otras variantes dialectales del español es cotidiano.

Dado lo anterior, las dos preguntas que intenta responder este artículo son: ¿cómo se manifiesta la variación fonética del fonema / b / en lectura en voz alta realizada por profesionales chilenos? ¿Existe alguna relación 
entre la zona geográfica de procedencia de los hablantes y la producción de variantes alofónicas del fonema /b/? A lo largo de este artículo, se intentará dar cuenta de estas problemáticas.

\section{Metodología}

\subsection{Sujetos de la muestra}

Los sujetos de la investigación fueron tomados de una muestra de habla nacional, correspondiente al proyecto FONDECYT Regular $\mathrm{N}^{\circ}$ 1120886, denominado "Descripción fonético-fonológica de un modelo de pronunciación enseñable del español culto de Chile". Este proyecto busca establecer un modelo de pronunciación para el español de Chile, basado en evidencias descriptivas. Para esto, el equipo investigador del proyecto tomó una muestra de habla en ocho ciudades del país: Iquique, La Serena, Valparaíso, Santiago, Concepción, Temuco, Coyhaique y Punta Arenas. El número final de sujetos ascendió a 250 informantes. A cada uno de ellos, se les pidió leer un texto ad hoc ${ }^{4}$, el cual contenía cada uno de los fonemas del español, en contextos fonéticos relevantes para la variante chilena; del mismo modo, se les sometió a una entrevista pauteada, para obtener habla espontánea. Las grabaciones de la lectura y de la entrevista fueron luego evaluadas por otros hablantes -chilenos nativos, mayores de 18 años, sin conocimientos conscientes de fonética-, quienes, utilizando una encuesta de apreciación subjetiva, en la que se les presentó una serie de extractos de las entrevistas y de las lectura, emitieron sus juicios sobre el habla y la pronunciación de los informantes, para luego poder calificarlos y catalogarlos como cultos o no.

Para el trabajo que ahora se expone, se tomaron los cuatro hablantes mejor evaluados de cada ciudad en la modalidad de lectura en voz alta ${ }^{5}$, por lo que la muestra que se analiza a continuación quedó constituida por 32 informantes.

4 Ver anexo.

5 Al respecto, Valdivieso, Merello y Candia opinan que "la lectura en voz alta es una actividad culturalmente muy relevante y frente a ella la comunidad parece adoptar la actitud más crítica" (1985, p. 89). Consecuentemente, se asume que los lectores deberán hacer gala de su mejor pronunciación para enfrentar esta tarea. 


\subsection{Corpus de análisis y variantes observadas}

En cuanto a las variantes, se rastrearon en la lectura todos los sonidos que correspondieran a realizaciones del fonema $/ \mathrm{b} /$, que coincidieran en el texto con los grafemas $\langle\mathrm{v}>\mathrm{o}\langle\mathrm{b}\rangle$. Las variantes que se pudieron establecer son las que se muestran en la tabla de la figura $\mathrm{N}^{\circ} 1$, que ofrece también imágenes ilustrativas de los articuladores externos implicados en la producción de la variante:

Figura $\mathrm{N}^{\mathrm{o}}$ 1: Variantes de / b / e imágenes representativas de su producción.

\begin{tabular}{|c|c|c|c|}
\hline Símbolo & Articulación & Ejemplo & Imagen \\
\hline$[\mathbf{U}]$ & $\begin{array}{c}\text { aproximante, } \\
\text { labiodental, sonoro, } \\
\text { oral }\end{array}$ & $<$ ignoraba $>$ & \\
\hline$[\beta]$ & $\begin{array}{l}\text { aproximante, } \\
\text { bilabial, sonoro, } \\
\text { oral }\end{array}$ & $<$ débil > & \\
\hline
\end{tabular}

\begin{tabular}{cc}
\hline oclusivo, bilabial, \\
sonoro, oral
\end{tabular}

fricativo,
labiodental,
sonoro, oral


A diferencia del trabajo de Sadowsky (2010), en esta investigación se han considerado separadamente los fonos [v] y [0], para determinar tanto el punto de articulación como el modo de articulación.

El conjunto de instancias fónicas de los alófonos de / b / en la lectura en voz alta constituyó el corpus de análisis. En total, se trabajó con 800 instancias de dichas variantes.

\subsection{Grabación y filmación de la muestra}

La lectura que cada uno de los informantes hizo del texto fue registrada digitalmente con una grabadora Tascam, modelo DR-40. Además, para poder observar el movimiento de los labios y la lengua, se encuadró y filmó la parte inferior del rostro de cada informante, con una filmadora Sony, modelo HDR-PJ230. A juicio del equipo de investigación, el tamaño discreto de los aparatos utilizados facilitó una mayor espontaneidad en el habla de los informantes.

\subsection{Análisis auditivo y visual}

El análisis del corpus fonético definido consideró dos etapas. En la primera de ellas, cada uno de los investigadores, por separado, realizó un análisis auditivo clásico para determinar la articulación de cada una de las instancias fónicas de /b/, registrando en una planilla sus observaciones, para luego contrastarlas con las de los otros investigadores. En varios casos, resultó imposible establecer un consenso respecto de la variante utilizada por el informante en la instancia en cuestión. Por eso, la segunda etapa del análisis consistió en la observación visual de las filmaciones de la lectura, para definir la zona, el modo y el punto de articulación de cada instancia de /b/. De este segundo análisis, constrastado también entre los investigadores, se da cuenta en este trabajo.

\section{Resultados}

A continuación, se presentan los resultados del análisis de la relación entre las principales variantes del sonido / b / y la zona de residencia de los sujetos que formulan cada una de las instancias evaluadas. Para realizar dicho análisis, se efectúa una agrupación de las ocho ciudades que poseen informantes en las cuatro zonas que considera Wagner (1998), esto es: Zona Norte: Iquique y la Serena; Zona Centro: Valparaíso y 
Santiago; Zona Sur: Concepción y Temuco; Zona Sur Austral: Coyhaique y Punta Arenas. Además, las variantes consideradas para dicho efecto son: [0], [ $\beta]$ y $[b]$, las cuales se asocian con las siguientes categorías numéricas: 1,2 , y 3, respectivamente, para efecto del procesamiento en el programa estadístico. Finalmente, hay que señalar que se descartaron las variantes con escasa frecuencia en la muestra, estas son: $[v],[\beta]$ y [Ø].

La tabla de la figura $\mathrm{N}^{\circ} 2$ muestra las frecuencias de las distintas variantes, presentes en el total de instancias, según la zona a la que pertenece el informante. En ella puede notarse que la variante [b] se distribuye de manera homogénea en las distintas zonas, mientras que la variante [v] se encuentra presente en mayor medida en la zona norte, contrario a lo que pasa con $[\beta]$ cuya frecuencia es más alta en las zonas sur y sur austral.

Figura $\mathrm{N}^{\mathrm{o}} 2$. Tabla de contingencia que representa la frecuencia de variantes por cada zona.

\begin{tabular}{lcccc}
\hline \multicolumn{5}{c}{ Variante } \\
\hline Zona & $\boldsymbol{v}(1)$ & $\beta_{\boldsymbol{\tau}}(2)$ & $\mathrm{b}(3)$ & Total \\
\hline Norte & 54 & 100 & 32 & 186 \\
\hline Centro & 54 & 107 & 32 & 193 \\
\hline Sur & 25 & 137 & 32 & 194 \\
\hline Sur Austral & 39 & 126 & 32 & 197 \\
\hline Total & 172 & 470 & 128 & 770 \\
\hline
\end{tabular}

Al realizar un análisis de dependencia, mediante el test Chi cuadrado, encontramos evidencia estadística (valor $\mathrm{p}=0,001$, que es menor a 0,05 , el valor crítico usual) para rechazar la hipótesis de independencia entre la variante y la zona a la que pertenecen los informantes. De esta manera, se puede concluir que, estadísticamente, existe algún tipo de relación entre ambas variables.

La tabla de la figura $\mathrm{N}^{\circ} 3$ muestra la manera en que se distribuyen las variantes según la zona de residencia de los informantes. Es así como se puede ver que la variante $\left[\beta_{\mathrm{T}}\right.$ ] es la que se pronuncia en un mayor porcentaje $(61 \%)$, contando todas las instancias pesquisadas. También, 
dicha tabla permite observar que en la zona sur un $71 \%$ de las instancias corresponden a la variante $\left[\beta_{\mathrm{r}}\right]$, alzándose como la zona donde se presenta en mayor proporción. Contrariamente, en esta misma zona la variante [0] se observa solo en un $13 \%$ de las instancias, siendo la variante que tiene menor presencia.

En la zona norte, la variante $\left[\beta_{\mathrm{r}}\right]$ también es la que se da en mayor proporción (54\%); sin embargo, dicho porcentaje es el más bajo de dicha variante, al observar el resto de las zonas. Contrariamente, la variante [ $\beta$ ] en esta zona presenta su porcentaje más alto (29\%). Algo muy similar ocurre en la zona centro, es decir, a pesar de que la variante $\left[\beta_{\mathrm{r}}\right]$ mantiene su importancia relativa -con un 55\%-, el porcentaje está muy próximo al de la zona norte, al igual que [], cuyo porcentaje (28\%) es muy similar al de dicha zona, pero más alto que el resto del país.

Figura No3. Perfiles fila, porcentaje respecto al total de filas.

\begin{tabular}{lcccc}
\hline \multirow{2}{*}{ Zona } & \multicolumn{3}{c}{ Variante } & \multirow{2}{*}{ Total } \\
\cline { 2 - 4 } & $\boldsymbol{U}(1)$ & $\beta_{\boldsymbol{r}}(2)$ & $\mathrm{b}(3)$ & \\
\hline Norte & $29 \%$ & $54 \%$ & $17 \%$ & $100 \%$ \\
\hline Centro & $28 \%$ & $55 \%$ & $17 \%$ & $100 \%$ \\
\hline Sur & $13 \%$ & $71 \%$ & $17 \%$ & $100 \%$ \\
\hline Sur Austral & $20 \%$ & $64 \%$ & $16 \%$ & $100 \%$ \\
\hline Total & $22 \%$ & $61 \%$ & $17 \%$ & $100 \%$ \\
\hline
\end{tabular}

La tabla de la figura $\mathrm{N}^{\circ} 4$ permite observar que, de todas las instancias en que se pronuncia la variante [U], la mayoría ocurre en la zona norte y centro, las que en conjunto reúnen al $62 \%$ de dicho sonido, muy por sobre el 38\% que suman, en conjunto, la zona sur y sur austral. Llama la atención la diferencia existente entre la zona sur y las zonas norte y centro, ya que en estas dos últimas se pronuncia el doble de dicha variante, en las instancias revisadas.

Para el caso de la variante $\left[\beta_{\mathrm{T}}\right]$, los resultados muestran que dicha variante se presenta en un mayor porcentaje en las zonas sur y sur austral, aunque las diferencias entre las zonas, no son tan altas como en el caso anterior. 
Finalmente, la variante [b] se distribuye de manera muy regular entre las distintas zonas.

Figura $\mathrm{N}^{\mathrm{o}} 4$. Perfiles columna, porcentaje respecto al total de columnas.

\begin{tabular}{lcccc}
\hline \multirow{2}{*}{ Zona } & \multicolumn{3}{c}{ Variante } & \multirow{2}{*}{ Total } \\
\cline { 2 - 4 } & $\boldsymbol{v}(1)$ & $\beta_{\boldsymbol{\tau}}(2)$ & $\mathrm{b}(3)$ & \\
\hline Norte & $31 \%$ & $21 \%$ & $25 \%$ & $24 \%$ \\
\hline Centro & $31 \%$ & $23 \%$ & $25 \%$ & $25 \%$ \\
\hline Sur & $15 \%$ & $29 \%$ & $25 \%$ & $25 \%$ \\
\hline Sur Austral & $23 \%$ & $27 \%$ & $25 \%$ & $26 \%$ \\
\hline Total & $100 \%$ & $100 \%$ & $100 \%$ & $100 \%$ \\
\hline
\end{tabular}

La figura $\mathrm{N}^{\circ} 5$ resume en un plano bidimensional los resultados de las tablas anteriores, al ejecutar un análisis de correspondencia simple, mediante una normalización simétrica. Dicho análisis permite explicar la relación que se manifiesta entre dos variables categóricas, a través de la proximidad que exista entre las distintas categorías o niveles (variantes $\mathrm{y}$ zonas, en este caso). En dicha figura, se puede apreciar que el eje $x$ (dimensión 1) realiza una separación notoria de las variantes, oponiendo claramente la [U] (1) con la [ $\left.\beta_{\mathrm{r}}\right]$ (2). Además, cada una de ellas aparece más próxima a algunas de las zonas definidas, de tal manera que [U] (1) está mucho más próxima a la zona norte y centro, y muy alejada de la zona sur, mientras que [ $\left.\beta_{\mathrm{r}}\right]$ (2), está más cerca de la zona sur y sur austral, que del resto del país.

Como resultado global, se puede mencionar que los datos muestran diferencias fonéticas importantes, cuando se analizan dichas variantes, las que están dadas principalmente por el fono [U], que se pronuncia con mayor frecuencia en la zona norte y centro, que en el resto del país.

También llama la atención el comportamiento que tiene el sonido [ $\left.\beta_{r}\right]$, que se da con mayor frecuencia en la zona sur, que en el resto del país. 
Figura No5. Gráfico bi-espacial entre las categorías de la variante y las zonas, producto del análisis de correspondencia.

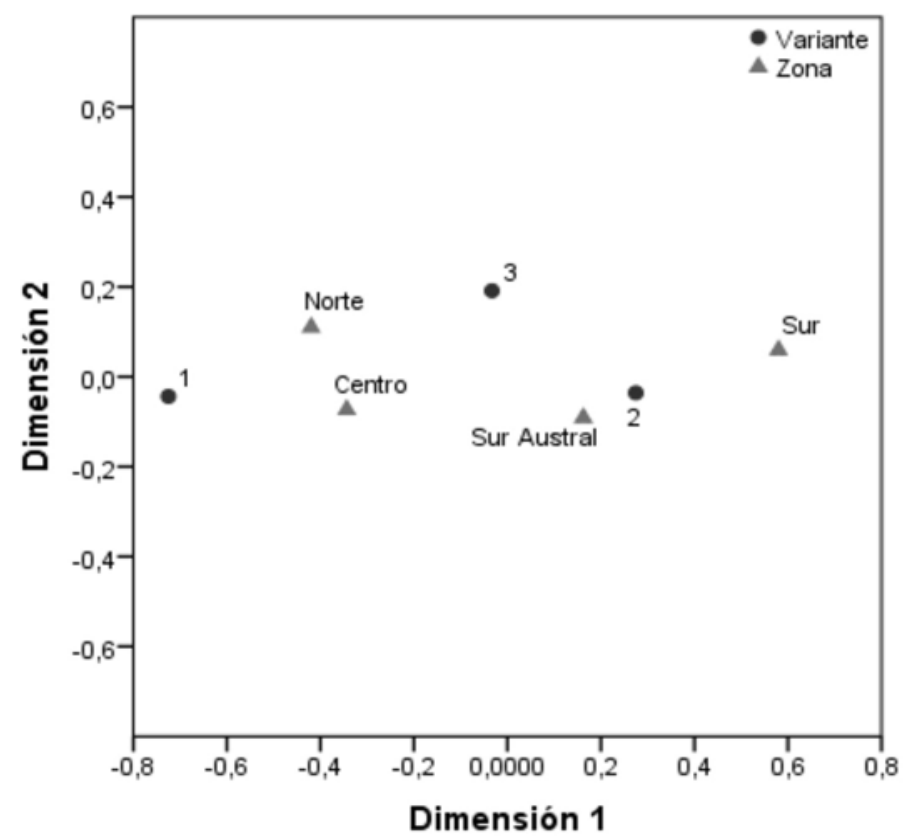

Nota: La categorías 1, 2 y 3 de la variable variante, corresponde a las variantes $[\mathcal{U}],\left[\beta_{\mathrm{r}}\right]$ y $[\mathrm{b}]$, respectivamente.

\section{Conclusiones y proyecciones}

A diferencia de lo establecido por Sadowsky (2010), Vergara (2011) y Vergara y Pérez, los hablantes profesionales de la muestra nacional no producen el fono fricativo labiodental sonoro oral [v] como variante de $/ \mathrm{b} /$. Es posible que esto se deba a que en dichos estudios, como ya se ha señalado, se discrimina el punto de articulación y no el modo. En todo caso, el hecho de que se haya manifestado en solo un informante en dos ocasiones no constituye más que un dato anecdótico en este estudio.

El fono oclusivo bilabial sonoro oral [b] mostró en los datos un alto grado de homogeneidad a nivel país (100\%), lo que era esperable, pues su pronunciación se encuentra fuertemente determinada por los contextos 
fonéticos en los que se observó: posición inicial absoluta y precedido de consonante nasal.

El fono aproximante bilabial sonoro oral $\left[\beta_{\mathrm{T}}\right]$ fue la variante más producida por los informantes en situación de lectura en voz alta. En efecto, obtuvo un $61 \%$ de ocurrencias a nivel país.

Por último, se observó variación diatópica en la producción de las variantes $[0]$ y $\left[\beta_{\uparrow}\right]$. Los resultados generales establecen que en la zona norte y centro se tiende a producir / $\mathrm{b} / \mathrm{como}$ [U], mientras que en las zona sur y sur austral la preferencia es pronunciar /b/ como [ $\beta_{\mathbf{r}}$. Estos resultados contravienen las observaciones de Sadowsky (2010), Vergara (2011) y Vergara y Pérez (2013) en cuanto a considerar la variante [v] como dominante en la pronunciacion chilena de $/ \mathrm{b} /$.

Como proyección de esta investigación, y considerando que el fenómeno del labiodentalismo en Chile muestra un avance importante en las zona norte y centro en los profesionales chilenos, en situación de lectura en voz alta, sería altamente conveniente explorar qué sucede en situación de entrevista semiespontánea. En otros términos, si en lectura en voz alta, actividad de alta relevancia social -como ya se ha señalado-, se manifiesta [0] de manera importante en algunas zonas geográficas del país, entonces este fenómeno debiera mostrar una tendencia igual o superior en situaciones elocutivas de menor control o autorregulación.

Por último, si se sigue la idea de Sadowsky (2010) en la cual declara que la pronunciación labiodental de / b / "en Chile sería un caso de innovación o cambio lingüístico" (p. 256), entonces una segunda proyección de este trabajo sería observar el fenómeno del labiodentalismo en hablantes de niveles socioculturales bajos, considerando que se reconoce que estos grupos de hablantes constituyen el sector sociolingüístico en el que primero se manifiesta la innovación o el cambio lingüístico. 


\section{Referencias bibliográficas}

Borland Delorme, K. (2004). La variación y distribución alofónica en el habla culta de Santiago de Chile. Onomázein, 10(2), 103-115.

Cartagena, N. (2002). Apuntes para la historia del español de Chile. Santiago: Cuadernos de la Academia Chilena de la Lengua.

Cepeda, G. (1991). Las consonantes de Valdivia. Valdivia: Imprenta América.

G. (1994). Las consonantes del español de Valdivia (Chile). Los procesos de reforzamiento y debilitamiento fonológico. Estudios Filológicos, 29, 39-61.

Cepeda, G. y Poblete, M.T. (1993). Retención y elisión de / $\beta$ / y / // en sufijos y morfemas radicales. Estudios Filológicos, $28,87-96$.

Lenz, R. (1893). Estudios Chilenos (Chilenische Student) II, III. PS 6; en Amado Alonso y Raimundo Lida (Eds.). El español en Chile (pp. 18-34). Buenos Aires: Universidad de Buenos Aires.

R. (1909). Fonética chilena i reglas para la transcripción de documentos en dialectos chileno. En Lenz, R. (Edit.) Programa de la Sociedad de Folklore Chileno (pp. 1824). Santiago: Imprenta y Encuadernación Lourdes.

Ortiz-Lira, H.; Saavedra. (2003). La fonética en Chile. Bibliografía analítica 1829-2003. Santiago: Phoné Libros.

Sadowsky, S. (2010). El alófono labiodental sonoro [v] del fonema / b / en el castellano de Concepción (Chile): una investigación exploratoria. Estudios de Fonética Experimental, XIX, 231-261.

Salas, A. (1996-1997). La lectura de noticias en la televisión chilena: modelo y norma en el fonetismo del castellano en Chile. Anuario de Lingüística Hispánica, 12/13, 819826. 
Soto-Barba, J. (2011). Variación consonántica en el habla urbana y rural de la provincia de Nuble. $R L A, 49,111-127$.

Valdivieso, H.; Merello, Z.; Candia, L. (1985). Fonética aplicada. Diagnóstico y tratamiento de deficiencias en la lectura en voz alta. $R L A, 23,89-106$.

Vergara, V. (2011). Producción de /v / como alófono de /b/ en niños prealfabetizados de la provincia de Concepción (Chile). Boletín de Lingüística, XXVIII, 35-36, 123-142.

Vergara, V; Pérez, H. E. (2013). Estudio dela incidencia dela representación gráfica (escritura) en la producción del alófono labiodental $[\mathrm{v}]$ del fonema /b/. Boletín de Filología, XLVIII, 2, 119-128.

Wigdorsky, L. (1978). Realización de algunos fonemas consonánticos en el castellano de Santiago. Informe preliminar. RLA, $16,53-60$.

Wagner, C. (1998). El Atlas lingüístico y etnográfico de Chile por regiones (ALECH). Estudios Filológicos, 33, 119-129. 


\section{ANEXO}

Texto para la lectura en voz alta. Se han destacado en negrita y subrayado las instancias gráficas donde se esperaba que el informante produjera algunas de las variantes de /b/ observadas en la investigación.

\section{INAMIBLE}

En la sala del juzgado el juez ya estaba en conocimiento de los hechos, después de leer el parte en voz alta, tras un breve instante de meditación, interrogó al reo. Don Andrés: ¿es verdad lo que se ha dicho aquí?, ¿qué tienes que alegar en tu defensa? Sin poder disimular su condición huasa, de abnegados esfuerzos diarios, la tímida respuesta del detenido fue igual a las anteriores. Sí usía, es verdad, pero yo ignoraba que estaba prohibido, la carne es débil. El magistrado hizo un gesto que parecía significar, sí conozco la cantinela, todos dicen lo mismo, y tomando la pluma escribió dos renglones al pie del parte policial, que enseguida devolvió al guardián, mientras decía con un habla un tanto chillona y fijando en el reo una magnánima mirada. Voy a darle dos años y un día de prisión, conmutables en ocho millones de multa. Tanta desproporción dejó al acusado trémulo, sintió el ritmo acelerado de su corazón, como si le corriera hielo por los huesos de la espalda, casi al borde del colapso, miró a su alrededor y murmuró dramáticamente con un ligero temblor de voz. Nunca habría pensado en tan absurda medida. ¿No habrá otra forma de pactar? El juez le advirtió que no replicara para no complicar todavía más su extrema situación. 